Weinberg recasts this equation in such a way that $H$ is a function of $\psi$ and its complex conjugate $\psi^{*}$. The approaches are equivalent if $H$ is linear in the product of $\psi$ and $\psi^{*}$. With higher powers of $\psi$ and $\psi^{*}$ involved, the equation becomes nonlinear. Weinberg maintains certain conditions that apply to normal quantum mechanics in investigating the implications of the generalized equation: for example, the hamiltonian must be 'homogeneous' so that systems separated from one another are treated properly.

Although this formulation has been designed to add nonlinearity as generally as possible, it has been pointed out that if the other postulates of quantum mechanics are left intact, it leads to violation of the second law of thermodynamics, which states that entropy always increases. Weinberg ${ }^{5}$ replies that the definition of entropy used in ordinary quantum mechanics cannot be taken over into its nonlinear generalization, so a new definition must be sought. Thus, at present there does not appear to be any reason to exclude nonlinear corrections on theoretical grounds.

In general one would expect no more than a small nonlinear correction to the normal hamiltonian to arise - large corrections would have become apparent long ago. The most simple choice for the correction in the case of a spin-half particle in a magnetic field yields a precession frequency $\omega_{\mathrm{p}}$ given by

$$
\omega_{\mathrm{p}}=\omega_{0}-4(\varepsilon / \hbar) \cos ^{2}(\theta / 2)
$$

where $\theta$ is the tipping angle and $\varepsilon$ measures the strength of the nonlinearity $\left(\omega_{0}\right.$ is the precession frequency normally predicted and $\hbar$ is Planck's constant).

The experimental system used for this investigation is a cloud of trapped and cooled beryllium ions ${ }^{6}$, exposed to two coherent bursts of microwave radiation separated by a long period of free precession (the Ramsey technique; see the News pages for more on Ramsey's Nobelprize winning work on atomic spectroscopy). The Penning ion trap, which uses a combination of static electric and magnetic fields to confine the ions to a small volume, contains of the order of $10,000{ }^{9} \mathrm{Be}^{+}$ ions. These are maintained at low temperatures (about $0.25 \mathrm{~K}$ ) by collisions with roughly $100,00{ }^{26} \mathrm{Mg}^{+}$ions held in the same trap. The ions are laser cooled (see my previous News and Views article ${ }^{7}$ ) by interaction with a laser beam tuned to a frequency just below their resonance line at 280 $\mathrm{nm}$. Each time an ion absorbs a photon from the beam it slows down by an amount corresponding to the absorbed photon momentum until sub-kelvin temperatures are reached. The $\mathrm{Be}^{+}$ions are then cooled 'sympathetically' by the cold $\mathrm{Mg}^{+}$ions. The lifetime of the ions in the trap is essentially infinite as the whole system is maintained in an ultra-high vacuum (at pressures below $10^{-13}$ atmospheres).

The transition investigated is a nuclear spin flip in the $\mathrm{Be}^{+}$ions, which is field independent to first order at $\mathrm{B}=0.82$ tesla and has a frequency of roughly $303 \mathrm{MHz}$. It is probed by irradiating the ion cloud with two coherent pulses (which would usually be $\pi / 2$ pulses, changing $\theta$ by $\pi / 2$ radians) separated by a period of the order of 100 seconds. It is only in an iontrap experiment that such long interaction times and low temperatures can be achieved and this is what makes the measurement so sensitive. After irradiating the ions with the $303 \mathrm{MHz}$ radiation, the number of ions to have made the transition is determined by measuring the population left in the initial state from the amount of fluorescence produced when the ions are irradiated by light at the $\mathrm{Be}^{+}$ resonance wavelength of $313 \mathrm{~nm}$.

The point of the experiment is to determine a difference in the precession frequency for different values of $\theta$, so the first coherent pulse used in the Ramsey probe is chosen to give either $\theta=\theta_{\mathrm{A}}=$ 1.02 radians or $\theta=\theta_{\mathrm{B}}=2.12$ radians. Using a conventional feedback system, the probe oscillator is locked to the ion's precession frequency with $\theta=\theta_{\mathrm{A}}$ for roughly 40 minutes, then with $\theta=\theta_{\mathrm{B}}$ for 40 minutes and so on. A sample plot of this locked frequency against time is shown in the figure and does not show any clear oscillation between two values as $\theta$ is changed, as would be expected if the precession frequency were changing with $\theta$ in the manner predicted.

The accuracy of the experiment is limited by the frequency reference available and the detected frequency shift, which is consistent with zero, is $3.8 \pm$ $8.3 \mu \mathrm{Hz}$ (the error corresponds to less than one complete cycle per day), yielding a value for $\varepsilon$ of $1.8 \pm 4.0 \mu \mathrm{Hz}$. Thus the maximum possible value of $\varepsilon$ is equivalent to an energy of $2.4 \times 10^{-20} \mathrm{eV}$, or less than 4 parts in $10^{27}$ of the binding energy per nucleon of the beryllium nucleus. This limit is $10^{5}$ times smaller than the previous one and can still be improved by roughly an order of magnitude with the present apparatus. Therefore, at the moment there is no reason to suppose that the framework of quantum mechanics shows nonlinearity at a significant level, though doubtless the search for such effects will continue.

Richard Thompson is in the Blackett Laboratory, Imperial College of Science, Technology and Medicine, Prince Consort Road, London SW7 2BZ, UK.

1. Weinberg, S. Phys. Rev. Lett. 62, 485-488 (1989)

2. Bollinger, J.J. et al. Phys. Rev. Lett. 63, 1031-1034 (1989).

3. Mandel, L. in Progress in Optics XIII (ed. Wolf, E.) 27-68 (North Holland, Amsterdam, 1976).

4. Peres, A. Phys, Rev. Lett. 63, 1114 (1989).

5. Weinberg, S. Phys. Rev. Lett. 63, 1115 (1989)

6. Bollinger, J.J. et al. Phys. Rev. Lett. 54, 1000-1003 (1985)

7. Thompson, R. Nature 335, 588-589 (1988).

\section{Stirrings of life}

LAST WEEK Daedalus invented his circularly polarized microwave reactor. It spins the polar molecules in a solution, driving them along like little propellers if they have the right asymmetry. The slow, diffusionlimited kinetics of chemical reactions is thus vastly accelerated. The chemistry of life, dominated by polar chiral molecules, should be particularly speeded up.

DREADCO's botanists are therefore constructing a novel 'Microtational forcing house' for plants. A powerful circularly polarized microwave field should speed their growth tremendously. Provided with extra warmth, intense light, concentrated nutrients and a carbon-rich atmosphere to fuel their furious growth, they should hustle from seed to maturity in a mere few hours or days. Indeed, when perfected, the new forcing house may be best laid out as a conveyor-belt production line, with seeds being continuously set on the belt at one end of the building and harvested as fully grown plants as they emerge at the other. Agriculture should be wonderfully compressed in space and time, releasing vast tracts of land for airports, motorways, leisure centres, theme parks and other progressive amenities.

The biochemical effects of circularly polarized microwaves on human and animal growth should be equally dramatic, and DREADCO's biologists are exploring them cautiously. Animal growth and metabolism should be directly accelerated. Microtational pig sties, cow sheds and hen houses should bring livestock to rapid maturity without the problems and side effects of hormone treatment. Medicine may gain as well. DREADCO's microtational sick bay could well speed up the healing of wounds, the regrowth of lost hair or nails and the defeat of infection. (On the other hand, bacterial multiplication may be accelerated even more.) Impatient body builders should also welcome a microtational gymnasium.

More revolutionary still, a microtational pregnancy smock might deliver an accelerated baby in far less than the traditional nine months. But a microtational cradle or nursery, to speed its subsequent physical progress, could have grave drawbacks. The long drawn out human childhood has evolved to give the growing child time to absorb the vast amount of information it will need as an adult. And learning, like all mental activities, depends ultimately on the speed of neural transmission - an ionic process unaffected by circularly polarized microwaves. So an accelerated child would gain rapidly in stature, but not in wisdom. He would reach physical adulthood while mentally still very childish and backward. He might also be biologically that much older and would grow old and die before his chronological time.

David Jones 